\title{
Habitual Miso (Fermented Soybean Paste) Consumption Is Associated with Glycemic Variability in Patients with Type 2 Diabetes: A Cross-Sectional Study
}

\author{
Fuyuko Takahashi ${ }^{1}$, Yoshitaka Hashimoto ${ }^{1, *} \mathbb{C}$, Ayumi Kaji ${ }^{1}$, Ryosuke Sakai ${ }^{1}$, Akane Miki ${ }^{1}$, Takuro Okamura ${ }^{1}$, \\ Noriyuki Kitagawa ${ }^{1,2}{ }^{(}$, Hiroshi Okada $\left.{ }^{1,3}{ }^{(}\right)$, Naoko Nakanishi ${ }^{1}$, Saori Majima ${ }^{1}$, Takafumi Senmaru ${ }^{1}$, \\ Emi Ushigome ${ }^{1}$, Masahide Hamaguchi ${ }^{1}\left[\right.$, Mai Asano ${ }^{1}$, Masahiro Yamazaki ${ }^{1}$ and Michiaki Fukui ${ }^{1}{ }^{1}$ \\ 1 Department of Endocrinology and Metabolism, Graduate School of Medical Science, \\ Kyoto Prefectural University of Medicine, 465, Kajii-cho, Kawaramachi-Hirokoji, Kamigyo-ku, \\ Kyoto 602-8566, Japan; fuyuko-t@koto.kpu-m.ac.jp (F.T.); kaji-a@koto.kpu-m.ac.jp (A.K.); \\ sakaryo@koto.kpu-m.ac.jp (R.S.); aknmk623@koto.kpu-m.ac.jp (A.M.); d04sm012@koto.kpu-m.ac.jp (T.O.); \\ nori-kgw@koto.kpu-m.ac.jp (N.K.); conti@koto.kpu-m.ac.jp (H.O.); naoko-n@koto.kpu-m.ac.jp (N.N.); \\ saori-m@koto.kpu-m.ac.jp (S.M.); semmarut@koto.kpu-m.ac.jp (T.S.); emis@koto.kpu-m.ac.jp (E.U.); \\ mhama@koto.kpu-m.ac.jp (M.H.); maias@koto.kpu-m.ac.jp (M.A.); masahiro@koto.kpu-m.ac.jp (M.Y.); \\ michiaki@koto.kpu-m.ac.jp (M.F.) \\ 2 Department of Diabetology, Kameoka Municipal Hospital, 1-1 Noda, Shinochoshino, Kameoka 621-8585, Japan \\ 3 Department of Diabetes and Endocrinology, Matsushita Memorial Hospital, 5-55 Sotojima-cho, \\ Moriguchi 570-8540, Japan \\ * Correspondence: y-hashi@koto.kpu-m.ac.jp; Tel.: +81-75-251-5505; Fax: +81-75-252-3721
}

Citation: Takahashi, F.; Hashimoto Y.; Kaji, A.; Sakai, R.; Miki, A.; Okamura, T.; Kitagawa, N.; Okada, H.; Nakanishi, N.; Majima, S.; et al. Habitual Miso (Fermented Soybean Paste) Consumption Is Associated with Glycemic Variability in Patients with Type 2 Diabetes: A

Cross-Sectional Study. Nutrients 2021, 13, 1488. https://doi.org/10.3390/ nu13051488

Academic Editor: Antonio Brunetti

Received: 2 March 2021

Accepted: 27 April 2021

Published: 28 April 2021

Publisher's Note: MDPI stays neutral with regard to jurisdictional claims in published maps and institutional affiliations.

Copyright: (c) 2021 by the authors. Licensee MDPI, Basel, Switzerland. This article is an open access article distributed under the terms and conditions of the Creative Commons Attribution (CC BY) license (https:// creativecommons.org/licenses/by/ $4.0 /)$.
Abstract: Glycemic control, including glycemic variability, is important for the prevention of diabetic vascular complications in patients with type 2 diabetes mellitus (T2DM). There was an association between miso soup intake and insulin resistance. However, the relationship between habitual miso consumption and glycemic control, including glycemic variability, in patients with T2DM remains unknown. We defined people without habitual miso consumption if they did not consume miso soup at all in a day. The average, standard deviation (SD), and coefficient of variation (CV), calculated as $\mathrm{CV}=(\mathrm{SD} /$ average $\mathrm{HbA} 1 \mathrm{c}) \times 100(\%)$, of hemoglobin $(\mathrm{Hb})$ A1c levels were evaluated. The proportions of habitual miso consumption of male and female were $88.1 \%$ and $82.3 \%$, respectively. The average (7.0 [6.4-7.5] vs. 7.3 [6.8-8.4] \%, $p=0.009)$, SD (0.21 [0.12-0.32] vs. 0.37 [0.20-0.72], $p=0.004)$, and CV (0.03 [0.02-0.04] vs. 0.05 [0.03-0.09], $p=0.005)$ of HbA1c levels in female with habitual miso consumption were lower than those of female without. Moreover, habitual miso consumption correlated with average $(\beta=-0.251, p=0.009), \operatorname{SD}(\beta=-0.175, p=0.016)$, and CV $(\beta=-0.185$, $p=0.022)$ of $\mathrm{HbA} 1 \mathrm{c}$ levels after adjusting for covariates. However, no association between habitual miso consumption and any glycemic parameters was shown among male. This study clarified the association between habitual miso consumption and good glycemic control, including glycemic variability, in female, but not in male.

Keywords: fermented soy foods; glycemic control; glycemic variability; miso; type 2 diabetes

\section{Introduction}

Glycemic control is important for the prevention of diabetic vascular complications in patients with type 2 diabetes mellitus (T2DM) [1,2]. Glycemic variability is important for preventing these complications, independent of the average glycemic control [3,4]. Hemoglobin $(\mathrm{Hb})$ A1c variability suggests insulin resistance and the presence of visceral adipose tissue [5]. A recent meta-analysis revealed that $\mathrm{HbA1c}$ variability was associated with renal disease, cardiovascular disease (CVD), and all-cause mortality in patients with T2DM and retinopathy [6]. Therefore, we need to focus not only on the mean glycemic control, but also on glycemic variability, especially HbA1c variability, as treatment targets in patients with T2DM. 
Miso, a traditional Japanese food made by fermenting soybeans, is very popular in Japan. It contains not only vegetable proteins, carbohydrates, and fats, but also minerals, vitamins, and microorganisms [7]. A previous review revealed that miso can inhibit the incidence of cancers in mice and rats [8]. Other studies have also shown the protective effect of miso on the incident of hypertension in Japanese people without hypertension $[9,10]$. Moreover, it has been reported that there is an association between habitual miso soup consumption and lower insulin resistance [11]. However, the relationship between habitual miso consumption and glycemic control in patients with T2DM remains unknown. Furthermore, a relationship between habitual miso consumption and glycemic variability in patients with T2DM has not been previously reported. Therefore, in this cross-sectional study of patients with T2DM, we researched the impact of habitual miso consumption on blood glucose control, including glycemic variability, in male and female.

\section{Method}

\subsection{Study Participants}

We commenced a prospective cohort study (KAMOGAWA-DM cohort study) in 2014, and it is currently ongoing [12]. Outpatients of the Kyoto Prefectural University of Medicine Hospital (Kyoto, Japan) and Kameoka Municipal Hospital (Kameoka, Japan) for diabetes was included in this cohort study. All included patients signed informed consent. This study was approved by the local research ethics committee (No. RBMR-E-466-6) and performed according to the Declaration of Helsinki. The inclusion criterion of this cross-sectional study was the ability to answer the questionnaires completely, including brief-type self-administered diet history questionnaire (BDHQ), from January 2016 to December 2018. The exclusion criteria were as follows: patients without T2DM, those with incomplete or inadequate responses to the questionnaire, and patients whose $\mathrm{HbA} 1 \mathrm{c}$ level was measured fewer than five times per year [13].

\subsection{Data Collection}

Family history of diabetes, exercise habits, smoking status, and duration of diabetes were assessed using a uniform questionnaire. Patients were categorized as smokers or non-smokers. Patients who regularly exercised were defined as those who regularly played any kind of sport $>1 \times /$ week.

Body mass index (BMI) and ideal body weight (IBW) [14] were calculated as weight (kg) divided by height squared $(\mathrm{m})^{2}$ and $22 \times(\text { height }[\mathrm{m}])^{2}$.

Venous blood was gathered from people with overnight fasting, and the levels of fasting blood sugar, triglycerides, high-density lipoprotein (HDL) cholesterol, uric acid, and creatinine $(\mathrm{Cr})$ were measured. Triglycerides to HDL cholesterol ratio was calculated as triglycerides ( $\mathrm{mmol} / \mathrm{L})$ divided by HDL cholesterol ( $\mathrm{mmol} / \mathrm{L})$ [15]. Estimated glomerular filtration rate (eGFR) was calculated: eGFR $\left(\mathrm{mL} / \mathrm{min} / 1.73 \mathrm{~m}^{2}\right)=194 \times \mathrm{Cr}^{-1.094} \times$ age $^{-0.287}$ $(\times 0.739$, if woman) [16]. HbA1c levels were estimated using high-performance liquid chromatography and expressed as NGSP units. Blood pressure was automatically measured using a HEM-906 device (OMRON, Kyoto, Japan) after the participants rested for $5 \mathrm{~min}$ in a quiet space. Furthermore, data on medications, including those for usage of insulin and antihypertensives, were collected from the patients' medical records. Patients with hypertension were defined as those with systolic/diastolic blood pressure $\geq 140 / 90 \mathrm{mmHg}$ and/or those using antihypertensive drugs.

\subsection{Definition of Glycemic Parameters}

We measured $\mathrm{HbA1c}$ at every visit. The $\mathrm{HbA} 1 \mathrm{c}$ levels, measured throughout the year, were obtained from electronic medical records. The average level, the standard deviation (SD), and the coefficient of variation (CV), defined as (SD/average HbA1c) $\times 100(\%)$, of $\mathrm{HbA} 1 \mathrm{c}$ were then assessed. 


\subsection{Data of Habitual Intake of Diet Intake}

A BDHQ was used to evaluate habitual food and nutrient intake in the previous month [17]. The specifications and validity of BDHQ were reported previously [17]. Data of the intake of energy, carbohydrates, proteins, fat, and fiber as well as miso soup and alcohol consumption were obtained using BDHQ. Frequency of miso soup intake was how often they consume miso soup per day; none, less than 1 cup, 1 cup, 2 cups, 3 cups, 4 cups, 5 cups, 6 or 7 cups, or 8 cups or more per day. We defined people without habitual miso consumption if they did not consume miso soup at all in a day [18].

\subsection{Statistical Analysis}

Data are shown as mean (SD), median [interquartile range] when the variables were skewed. A $p$-value of $<0.05$ was considered to be statistically significant.

Participants were categorized into two groups based on habitual miso consumption. Because the characteristics and dietary intakes were different between sex, the data were analyzed male and female, separately. Evaluation of normal distribution was performed by Shapiro-wilk normality test. Student's $t$-test or Mann-Whitney U test for continuous variables and chi-square test for categorical variables was used for evaluation of the differences. Evaluation of correlation was assessed by Pearson's correlation coefficient. Because the miso soup intake was a skewed variable, logarithmic transformation was done, before performing the investigation of the relationship of glycemic variability and log (miso soup intake +1$)$.

To investigate the effect of habitual miso consumption on glycemic parameters, including average, $\mathrm{SD}$, and $\mathrm{CV}$ of $\mathrm{HbA1c}$, multiple regression analyses was used, adjusting for potential cofounders; age, BMI, duration of diabetes, average HbA1c, insulin treatment [19], exercise habit, smoking status, intake of energy [20], intake of carbohydrate, and intake of dietary fiber [21]. Statistical analyses were performed by JMP software (version 13.2; SAS Institute Inc., Cary, NC, USA) and EZR (Saitama Medical Center, Jichi Medical University, Saitama, Japan) [22].

\section{Results}

This study included 523 patients (276 male, 247 female). Among them, 121 patients (57 male and 64 female) with no data on BDHQ, 40 patients (20 male and 20 female) without T2DM, and 72 patients ( 39 male and 53 female) whose HbA1c levels were measured fewer than five times per year were excluded. Therefore, 290 patients (160 male and 130 female) were included in the study (Figure 1).

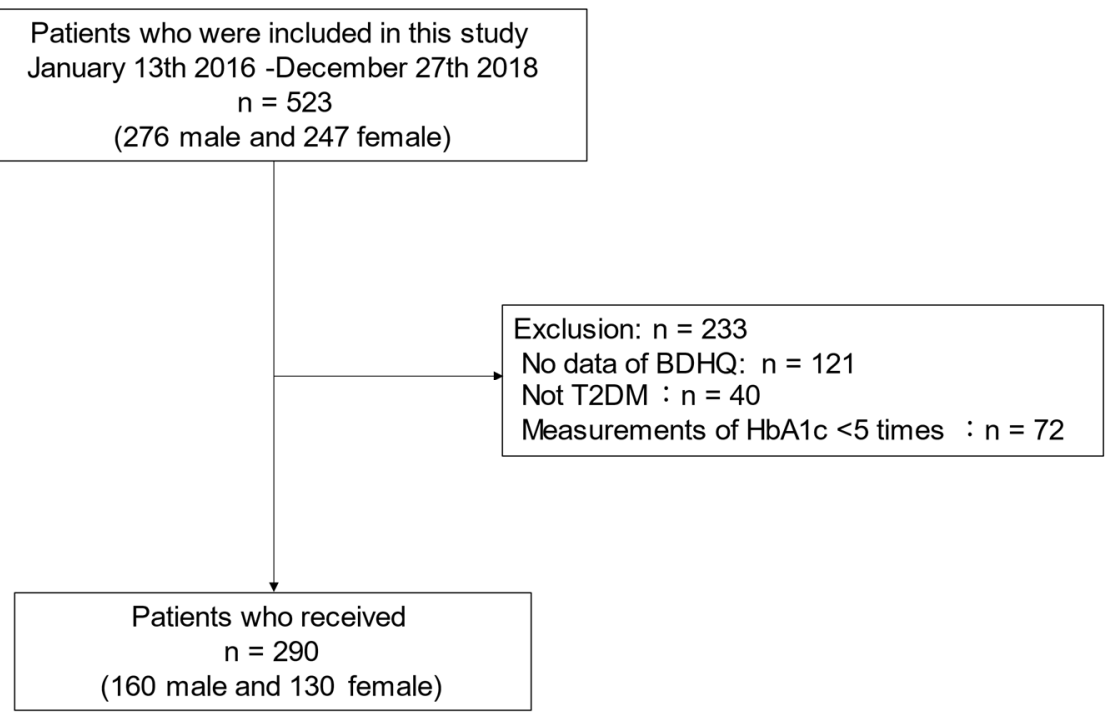

Figure 1. Inclusion and exclusion flow. 
Table 1 represent the baseline clinical characteristics of the study participants. Median age and BMI were 68.0 [63.0-74.0] years and $23.5[21.6-25.8] \mathrm{kg} / \mathrm{m}^{2}$ in male and $68.0[62.0-73.8]$ years and $23.6[21.3-26.3] \mathrm{kg} / \mathrm{m}^{2}$ in female. The average, SD, and CV of HbA1c were 7.0 [6.6-7.5] \%, 0.21 [0.14-0.33], and 0.03 [0.02-0.05] in male and 7.0 [6.5-7.6] \%, $0.22[0.13-0.37]$, and $0.03[0.02-0.05]$ in female, respectively.

Table 1. Clinical characteristic of study patients.

\begin{tabular}{|c|c|c|c|c|}
\hline & $\begin{array}{c}\text { All } \\
n=290\end{array}$ & $\begin{array}{c}\text { Male } \\
n=160\end{array}$ & $\begin{array}{l}\text { Female } \\
n=130\end{array}$ & $p$ \\
\hline Age, years & $68.0[62.3-74.0]$ & $68.0[63.0-74.0]$ & $68.0[62.0-73.8]$ & 0.491 \\
\hline Diseases duration of diabetes, years & $13.0[7.0-20.0]$ & $14.0[8.0-21.0]$ & $12.5[5.3-18.0]$ & 0.110 \\
\hline Family history of diabetes $(-/+)$ & $155 / 135$ & $92 / 68$ & $63 / 67$ & 0.157 \\
\hline Height, cm & $161.0(9.2)$ & $167.2(6.5)$ & $153.3(5.4)$ & $<0.001$ \\
\hline Body weight, $\mathrm{kg}$ & $61.0[54.8-68.7]$ & $65.3[59.2-72.6]$ & $55.7[51.0-61.8]$ & $<0.001$ \\
\hline Body mass index, $\mathrm{kg} / \mathrm{m}^{2}$ & $23.5[21.4-26.0]$ & $23.5[21.6-25.8]$ & $23.6[21.3-26.3]$ & 0.481 \\
\hline Systolic BP, mmHg & $132.0[121.0-144.0]$ & $132.0[122.0-144.0]$ & $132.0[120.0-143.0]$ & 0.748 \\
\hline Diastolic BP, $\mathrm{mmHg}$ & $77.5[71.0-85.0]$ & $80.0[71.0-86.0]$ & $76.0[70.0-82.0]$ & 0.055 \\
\hline Antihypertensive drugs $(-/+)$ & $123 / 167$ & $66 / 94$ & $57 / 73$ & 0.745 \\
\hline Presence of hypertension $(-/+)$ & $94 / 196$ & $54 / 106$ & $40 / 90$ & 0.679 \\
\hline Insulin $(-/+)$ & $211 / 79$ & $118 / 42$ & $93 / 37$ & 0.773 \\
\hline Habit of smoking $(-/+)$ & $246 / 44$ & $126 / 34$ & $120 / 10$ & 0.002 \\
\hline Habit of exercise $(-/+)$ & $140 / 150$ & $76 / 84$ & $64 / 66$ & 0.861 \\
\hline Average $\mathrm{HbA} 1 \mathrm{c}, \mathrm{mmol} / \mathrm{mol}$ & $53.3[48.3-58.9]$ & $53.5[49.0-58.5]$ & $53.2[48.0-59.1]$ & 0.544 \\
\hline Average $\mathrm{HbA1c}, \%$ & $7.0[6.6-7.5]$ & $7.0[6.6-7.5]$ & $7.0[6.5-7.6]$ & 0.544 \\
\hline $\mathrm{SD}$ of $\mathrm{HbA1c}$ & $0.21[0.14-0.34]$ & $0.21[0.14-0.33]$ & $0.22[0.13-0.37]$ & 0.899 \\
\hline $\mathrm{CV}$ of $\mathrm{HbA} 1 \mathrm{c}$ & $0.03[0.02-0.05]$ & $0.03[0.02-0.05]$ & $0.03[0.02-0.05]$ & 0.962 \\
\hline Plasma glucose, $\mathrm{mmol} / \mathrm{L}$ & $7.7[6.5-9.3]$ & $7.9[6.8-10.0]$ & $7.5[6.2-8.9]$ & 0.042 \\
\hline Creatinine, umol/L & $64.5[55.0-80.4]$ & $74.7[63.6-88.4]$ & $55.7[49.5-63.4]$ & $<0.001$ \\
\hline $\mathrm{eGFR}, \mathrm{mL} / \mathrm{min} / 1.73 \mathrm{~m}^{2}$ & $71.8[59.0-82.5]$ & $71.6[57.4-83.6]$ & $72.5[60.9-81.8]$ & 0.752 \\
\hline Uric acid, $\mathrm{mmol} / \mathrm{L}$ & $297.4[255.8-350.9]$ & $3188.2[273.6-364.3]$ & $279.6[243.9-321.2]$ & $<0.001$ \\
\hline Triglycerides, mmol/L & $1.2[0.9-1.9]$ & $1.3[0.9-1.9]$ & $1.2[0.9-1.7]$ & 0.698 \\
\hline HDL cholesterol, $\mathrm{mmol} / \mathrm{L}$ & $1.5[1.3-1.8]$ & $1.4[1.2-1.7]$ & $1.6[1.3-1.8]$ & 0.005 \\
\hline $\begin{array}{c}\text { Triglycerides/HDL } \\
\text { cholesterol, } \mathrm{mmol} / \mathrm{mmol}\end{array}$ & $0.8[0.5-1.4]$ & $0.8[0.5-1.5]$ & $0.8[0.5-1.2]$ & 0.166 \\
\hline
\end{tabular}

Number (absence [-]/presence [+]), mean (standard deviation) or median [interquartile range] was used for expression of data. Chi-square test, Student's t test, or Mann-Whitney U test was used for evaluation of the difference between group. CV, coefficient of variation; BP, blood pressure; eGFR, estimated glomerular filtration rate; HDL, high-density lipoprotein; and SD, standard deviation.

BDHQ, brief-type self-administered diet history questionnaire; T2DM, type 2 diabetes mellites.

Table 2 shows the results of dietary intake data. The percentage of habitual miso consumption was $88.1 \%(n=141 / 160)$ and $82.3 \%(n=107 / 130)$ in male and female, respectively.

Table 3 represents the clinical characteristics of patients based on habitual miso consumption or not. Among females, the average $(p=0.009), \operatorname{SD}(p=0.004)$, and CV $(p=0.005)$ of $\mathrm{HbA1c}$ were lower in patients with habitual miso consumption than in those without it. Fasting blood sugar in females with habitual miso consumption was lower than in those without it $(p=0.034)$. The percentage of hypertension was lower in females with habitual miso consumption than in females without $(p=0.023)$. However, there was no association between habitual miso consumption and clinical characteristics in males. 
Table 2. Habitual diet intake of study participants.

\begin{tabular}{|c|c|c|c|c|}
\hline & $\begin{array}{c}\text { All } \\
n=290\end{array}$ & $\begin{array}{c}\text { Male } \\
n=160\end{array}$ & $\begin{array}{l}\text { Female } \\
n=130\end{array}$ & $p$ \\
\hline Intake of total energy, $\mathrm{kcal} /$ day & 1658.2 [1338.1-2061.3] & 1813.4 [1541.5-2180.7] & 1483.4 [1188.8-1779.4] & $<0.001$ \\
\hline Energy intake, kcal/IBW/day & $29.2[23.9-35.8]$ & $30.0[25.2-36.2]$ & $28.1[22.0-34.6]$ & 0.137 \\
\hline Intake of total protein, $\mathrm{g} /$ day & $66.8[55.5-85.1]$ & $71.5[57.3-88.2]$ & $62.4[50.7-82.4]$ & 0.013 \\
\hline Intake of protein, $\mathrm{g} / \mathrm{IBW} /$ day & $1.2[0.9-1.5]$ & $1.2[0.9-1.4]$ & $1.2[1.0-1.6]$ & 0.116 \\
\hline Intake of protein per energy, $\%$ & $16.2[14.3-18.9]$ & $15.4[13.5-17.7]$ & $17.6[15.4-20.2]$ & $<0.001$ \\
\hline Intake of animal protein, $\mathrm{g} /$ day & $40.8[30.4-54.0]$ & $41.4[31.4-56.0]$ & $38.5[29.5-53.0]$ & 0.152 \\
\hline Intake of animal protein, $\mathrm{g} / \mathrm{IBW} /$ day & $0.7[0.5-1.0]$ & $0.7[0.5-0.9]$ & $0.8[0.5-1.0]$ & 0.089 \\
\hline Intake of vegetable protein, g/day & $27.2[21.3-32.9]$ & $29.0[23.4-35.7]$ & $24.9[20.1-30.5]$ & $<0.001$ \\
\hline $\begin{array}{l}\text { Intake of vegetable protein, } \\
\text { g/IBW/day }\end{array}$ & $0.5[0.4-0.6]$ & $0.5[0.4-0.6]$ & $0.5[0.4-0.6]$ & 0.777 \\
\hline Animal/vegetable protein intake ratio & $1.5[1.1-2.0]$ & $1.5[1.1-1.9]$ & $1.6[1.2-2.1]$ & 0.141 \\
\hline Intake of total fat, g/day & $51.5[40.4-67.9]$ & $55.8[44.1-70.2]$ & $47.6[38.2-60.6]$ & $<0.005$ \\
\hline Intake of fat intake, $\mathrm{g} / \mathrm{IBW} /$ day & $0.9[0.7-1.2]$ & $0.9[0.7-1.1]$ & $0.9[0.7-1.2]$ & 0.817 \\
\hline Intake of fat per energy intake, $\%$ & $28.8(6.5)$ & $27.8(6.6)$ & $29.9(6.3)$ & 0.008 \\
\hline Intake of total carbohydrate, $\mathrm{g}$ / day & $214.5[162.2-256.0]$ & $231.5[184.8-275.1]$ & 188.9 [146.1-229.2] & $<0.001$ \\
\hline Intake of carbohydrate, $\mathrm{g} / \mathrm{IBW} /$ day & $3.7[2.9-4.5]$ & $3.8[3.1-4.6]$ & $3.6[2.7-4.4]$ & 0.279 \\
\hline $\begin{array}{c}\text { Intake of carbohydrate per energy } \\
\text { intake, } \%\end{array}$ & $50.7(8.9)$ & $50.4(9.2)$ & $51.0(8.5)$ & 0.557 \\
\hline Intake of dietary fiber, $\mathrm{g} /$ day & $11.2[8.8-15.2]$ & $11.1[8.7-15.6]$ & $11.4[8.8-15.1]$ & 0.772 \\
\hline Carbohydrate/fiber ratio & $18.6[14.3-24.0]$ & $20.4[15.9-25.4]$ & $16.4[14.0-20.5]$ & $<0.001$ \\
\hline Alcohol consumption, g/day & $0.0[0.0-3.6]$ & $1.7[0.0-16.7]$ & $0.0[0.0-0.0]$ & $<0.001$ \\
\hline Habitual miso consumption $(-/+)$ & $42 / 248$ & $19 / 141$ & $23 / 107$ & 0.218 \\
\hline Miso soup intake, $\mathrm{g} /$ day & $108.0[55.4-1138.6]$ & 110.9 [62.4-138.6] & $96.0[48.0-120.0]$ & $<0.001$ \\
\hline
\end{tabular}

Number (absence [-]/presence [+]), mean (standard deviation) or median [interquartile range] was used for expression of data. Chi-square test, Student's t test, or Mann-Whitney U test was used for evaluation of the difference between group. IBW, ideal body weight.

Table 3. Clinical characteristics according to habitual miso consumption.

\begin{tabular}{|c|c|c|c|c|c|c|}
\hline & \multicolumn{3}{|c|}{$\begin{array}{c}\text { Male } \\
n=160\end{array}$} & \multicolumn{3}{|c|}{$\begin{array}{l}\text { Female } \\
n=130\end{array}$} \\
\hline & $\begin{array}{c}\text { Habitual Miso } \\
\text { Consumption }(-) \\
\qquad n=19\end{array}$ & $\begin{array}{c}\text { Habitual Miso } \\
\text { Consumption (+) } \\
\quad n=141\end{array}$ & $p$ & $\begin{array}{c}\text { Habitual Miso } \\
\text { Consumption }(-) \\
\qquad n=23\end{array}$ & $\begin{array}{c}\text { Habitual Miso } \\
\text { Consumption (+) } \\
\quad n=107\end{array}$ & $p$ \\
\hline Age, years & $68.0[64.0-79.5]$ & $68.0[63.0-74.0]$ & 0.419 & $67.0[61.0-73.0]$ & $68.0[62.5-73.5]$ & 0.548 \\
\hline Diseases duration of diabetes, years & $16.0[12.5-27.0]$ & $13.0[8.0-21.0]$ & 0.087 & $17.0[8.0-22.0]$ & $12.0[4.5-18.0]$ & 0.035 \\
\hline Family history of diabetes $(-/+)$ & $11 / 8$ & $81 / 60$ & 1.000 & $9 / 14$ & $54 / 53$ & 0.449 \\
\hline Height, $\mathrm{cm}$ & $165.4(6.8)$ & $167.4(6.5)$ & 0.206 & $152.7(5.1)$ & $153.5(5.5)$ & 0.530 \\
\hline Body weight, $\mathrm{kg}$ & $68.0[57.7-73.3]$ & $65.0[59.8-72.0]$ & 0.941 & $58.0[54.6-66.0]$ & $55.1[49.6-60.6]$ & 0.046 \\
\hline Body mass index, $\mathrm{kg} / \mathrm{m}^{2}$ & $24.2(2.8)$ & $23.7(3.3)$ & 0.529 & $24.8[23.2-29.0]$ & $23.4[21.1-26.1]$ & 0.052 \\
\hline Systolic BP, mmHg & $128.0[114.0-137.0]$ & $133.0[122.0-144.0]$ & 0.148 & $135.0[132.0-143.0]$ & $131.0[119.0-143.0]$ & 0.113 \\
\hline Diastolic BP, mmHg & $75.5(11.5)$ & $79.9(11.0)$ & 0.099 & $74.0[68.5-78.0]$ & $76.0[71.0-84.0]$ & 0.224 \\
\hline Antihypertensive drugs $(-/+)$ & $5 / 14$ & $61 / 80$ & 0.246 & $7 / 16$ & $50 / 57$ & 0.231 \\
\hline Presence of hypertension $(-/+)$ & $4 / 15$ & $50 / 91$ & 0.323 & $2 / 21$ & $38 / 69$ & 0.023 \\
\hline Insulin $(-/+)$ & $17 / 2$ & $101 / 40$ & 0.167 & $13 / 10$ & $80 / 27$ & 0.132 \\
\hline Habit of smoking $(-/+)$ & $15 / 4$ & $111 / 30$ & 1.000 & $20 / 3$ & $100 / 7$ & 0.528 \\
\hline Habit of exercise $(-/+)$ & $11 / 8$ & $65 / 76$ & 0.470 & $11 / 12$ & $53 / 54$ & 1.000 \\
\hline Average $\mathrm{HbA} 1 \mathrm{c}, \mathrm{mmol} / \mathrm{mol}$ & $55.0[46.2-60.6]$ & $53.4[49.0-58.3]$ & 0.947 & $56.7[50.4-68.7]$ & $52.5[46.9-58.1]$ & 0.009 \\
\hline Average $\mathrm{HbA1c}, \%$ & $7.2[6.4-7.7]$ & $7.0[6.6-7.5]$ & 0.947 & $7.3[6.8-8.4]$ & $7.0[6.4-7.5]$ & 0.009 \\
\hline $\mathrm{SD}$ of $\mathrm{HbA} 1 \mathrm{c}$ & $0.27[0.14-0.38]$ & $0.20[0.14-0.32]$ & 0.603 & $0.37[0.20-0.72]$ & $0.21[0.12-0.32]$ & 0.004 \\
\hline $\mathrm{CV}$ of $\mathrm{HbA} 1 \mathrm{c}$ & $0.04[0.02-0.05]$ & $0.03[0.02-0.05]$ & 0.572 & $0.05[0.03-0.09]$ & $0.03[0.02-0.04]$ & 0.005 \\
\hline Fasting blood sugar, mmol/L & $7.7[6.2-9.1]$ & $7.9[6.9-10.1]$ & 0.256 & $8.4[7.0-10.6]$ & $7.3[6.2-8.5]$ & 0.034 \\
\hline Creatinine, umol/L & $82.2[67.6-87.5]$ & $73.4[63.6-88.4]$ & 0.296 & $55.7[49.9-69.8]$ & $55.7[49.5-63.2]$ & 0.784 \\
\hline $\mathrm{eGFR}, \mathrm{mL} / \mathrm{min} / 1.73 \mathrm{~m}^{2}$ & $67.8(19.5)$ & $71.4(20.4)$ & 0.470 & $69.1(24.6)$ & $71.7(16.4)$ & 0.532 \\
\hline Uric acid, mmol/L & $327.1[291.5-348.0]$ & $315.2[267.7-368.8]$ & 0.710 & $285.0(52.0)$ & $287.0(63.9)$ & 0.887 \\
\hline Triglycerides, mmol/L & $1.1[0.8-1.8]$ & $1.3[0.9-1.9]$ & 0.718 & $1.3[0.9-1.8]$ & $1.2[0.9-1.6]$ & 0.481 \\
\hline HDL cholesterol, $\mathrm{mmol} / \mathrm{L}$ & $1.4[1.3-1.5]$ & $1.4[1.2-1.7]$ & 0.677 & $1.5[1.4-1.7]$ & $1.6[1.3-1.8]$ & 0.850 \\
\hline $\begin{array}{c}\text { Triglycerides/HDL } \\
\text { cholesterol, } \mathrm{mmol} / \mathrm{mol}\end{array}$ & $0.8[0.6-1.4]$ & $0.8[0.5-1.6]$ & 0.924 & $0.9[0.6-1.2]$ & $0.7[0.5-1.2]$ & 0.426 \\
\hline
\end{tabular}

Number (absence [-]/presence [+]), mean (standard deviation) or median [interquartile range] was used for expression of data. Chi-square test, Student's $\mathrm{t}$ test, or Mann-Whitney U test was used for evaluation of the difference between group. BP, blood pressure; $\mathrm{CV}$, coefficient of variation; eGFR, estimated glomerular filtration rate; HDL, high-density lipoprotein; and SD, standard deviation. 
Habitual dietary intake based on the presence of habitual miso consumption are shown in Table 4. The intakes of energy $(p<0.001)$, protein $(p<0.001)$, animal protein $(p=0.010)$, vegetable protein $(p<0.001)$, fat $(p=0.031)$, and dietary fiber $(p<0.001)$ in females with habitual miso consumption were higher than those in females without it. In contrast, there was no difference in dietary habits between male with and without habitual miso consumption.

Table 4. Habitual diet intake according to habitual miso consumption.

\begin{tabular}{|c|c|c|c|c|c|c|}
\hline & \multicolumn{3}{|c|}{$\begin{array}{c}\text { Male } \\
n=160\end{array}$} & \multicolumn{3}{|c|}{$\begin{array}{l}\text { Female } \\
n=130\end{array}$} \\
\hline & $\begin{array}{c}\text { Habitual Miso } \\
\text { Consumption (-) } \\
n=19\end{array}$ & $\begin{array}{c}\text { Habitual Miso } \\
\text { Consumption (+) } \\
\quad n=141\end{array}$ & $p$ & $\begin{array}{c}\text { Habitual Miso } \\
\text { Consumption (-) } \\
n=23\end{array}$ & $\begin{array}{c}\text { Habitual Miso } \\
\text { Consumption (+) } \\
n=107\end{array}$ & $p$ \\
\hline Intake of total energy, $\mathrm{kcal} / \mathrm{day}$ & $\begin{array}{c}1616.3 \\
{[1390.6-2014.2]}\end{array}$ & $\begin{array}{c}1832.2 \\
{[1588.0-2192.8]}\end{array}$ & 0.148 & $\begin{array}{c}1245.5 \\
{[930.3-1455.9]}\end{array}$ & $\begin{array}{c}1574.0 \\
{[1207.1-1811.7]}\end{array}$ & $<0.001$ \\
\hline Energy intake, kcal/IBW/day & $27.3[22.2-33.1]$ & $30.3[25.5-36.3]$ & 0.311 & $24.9[18.8-27.7]$ & $29.4[23.2-36.1]$ & $<0.001$ \\
\hline Intake of total protein, $\mathrm{g} /$ day & $66.8[53.3-83.3]$ & $71.7[57.4-88.2]$ & 0.516 & $47.3[35.7-64.0]$ & $66.0[54.9-84.2]$ & $<0.001$ \\
\hline Intake of protein, g/IBW / day & $1.1[0.9-1.4]$ & $1.2[0.9-1.4]$ & 0.558 & $0.9[0.7-1.2]$ & $1.3[1.0-1.7]$ & $<0.001$ \\
\hline Intake of protein per energy, $\%$ & $15.3[14.4-18.5]$ & 15.5 [13.5-17.7] & 0.513 & $17.0(4.0)$ & $18.1(3.4)$ & 0.189 \\
\hline Intake of animal protein, g/day & $38.8[32.6-51.2]$ & $41.5[31.5-56.9]$ & 0.685 & $32.8[19.9-41.4]$ & $40.8[30.3-54.1]$ & 0.011 \\
\hline $\begin{array}{l}\text { Intake of animal protein, } \\
\text { g/IBW/day }\end{array}$ & $0.6[0.6-0.9]$ & $0.7[0.5-0.9]$ & 0.833 & $0.6[0.4-0.8]$ & $0.8[0.6-1.0]$ & 0.010 \\
\hline Intake of vegetable protein, $\mathrm{g} /$ day & $27.8(10.2)$ & $30.0(8.8)$ & 0.323 & $18.2[13.7-22.0]$ & $26.6[21.3-30.9]$ & $<0.001$ \\
\hline $\begin{array}{l}\text { Intake of vegetable protein, } \\
\text { g/IBW/day }\end{array}$ & $0.5(0.2)$ & $0.5(0.1)$ & 0.500 & $0.4[0.3-0.4]$ & $0.5[0.4-0.6]$ & $<0.001$ \\
\hline $\begin{array}{c}\text { Animal/vegetable protein intake } \\
\text { ratio }\end{array}$ & $1.4[1.1-2.1]$ & $1.5[1.1-1.9]$ & 0.704 & $1.6[1.1-2.2]$ & $1.6[1.2-2.1]$ & 0.860 \\
\hline Intake of total fat, g/day & $65.9[42.4-73.1]$ & $55.2[44.5-69.4]$ & 0.299 & $42.4[31.2-49.7]$ & $48.6[39.5-63.8]$ & 0.020 \\
\hline Intake of fat intake, g/IBW/day & $1.0[0.8-1.2]$ & $0.9[0.7-1.1]$ & 0.213 & $0.8[0.6-0.9]$ & $0.9[0.7-1.3]$ & 0.031 \\
\hline Intake of fat per energy intake, $\%$ & $32.8[27.0-35.8]$ & $27.1[24.0-30.5]$ & 0.003 & $30.8(7.4)$ & $29.7(6.1)$ & 0.456 \\
\hline Intake of total carbohydrate, g/day & $203.5[169.9-238.0]$ & $234.6[186.2-280.0]$ & 0.059 & $154.3[122.6-190.1]$ & $196.1[151.9-238.0]$ & 0.001 \\
\hline $\begin{array}{l}\text { Intake of carbohydrate, } \\
\text { g/IBW/day }\end{array}$ & $3.4[2.8-4.0]$ & $3.8[3.1-4.7]$ & 0.121 & $3.1[2.4-3.6]$ & $3.7[2.9-4.7]$ & 0.002 \\
\hline $\begin{array}{c}\text { Intake of carbohydrate per energy } \\
\text { intake, } \%\end{array}$ & $49.3(9.5)$ & $50.5(9.2)$ & 0.586 & $50.9(9.9)$ & $51.0(8.2)$ & 0.929 \\
\hline Intake of dietary fiber intake, g/day & $10.3[7.6-13.5]$ & $11.4[8.8-15.8]$ & 0.189 & $8.0[5.5-9.4]$ & $12.1[9.2-15.3]$ & $<0.001$ \\
\hline Alcohol consumption, g/day & $0.0[0.0-0.6]$ & $2.8[0.0-17.6]$ & 0.005 & $0.0[0.0-0.0]$ & $0.0[0.0-0.0]$ & 0.973 \\
\hline
\end{tabular}

Number (absence [-]/presence [+]), mean (standard deviation) or median [interquartile range] was used for expression of data. Chi-square test, Student's t test, or Mann-Whitney U test was used for evaluation of the difference between group. IBW, ideal body weight.

The correlation between the log (miso soup intake +1 ) and average, SD, or CV of $\mathrm{HbA1c}$ were investigated. In females, the $\log ($ miso soup intake +1$)$ was correlated with average $(r=-0.290, p<0.001), \mathrm{SD}(r=-0.316, p<0.001)$ and CV $(r=-0.289, p<0.001)$ of $\mathrm{HbA1c}$. On the other hand, the $\log$ (miso soup intake +1 ) was not correlated with average $(r=-0.079, p=0.322), \mathrm{SD}(r=-0.034, p=0.667)$ and $\mathrm{CV}(r=-0.013, p=0.874)$ of $\mathrm{HbA} 1 \mathrm{c}$ in males.

To examine the association between habitual miso consumption and glycemic parameters, we performed multiple regression analyses (Table 5). In females, habitual miso consumption was related to the average $(\beta=-0.251, p=0.009), \operatorname{SD}(\beta=-0.175, p=0.016)$, and $\mathrm{CV}(\beta=-0.185, p=0.022)$ of $\mathrm{HbA1}$ c levels after adjusting for covariates. However, habitual miso consumption was not associated with the average, $\mathrm{SD}$, and $\mathrm{CV}$ of $\mathrm{HbA} 1 \mathrm{c}$ levels in males. 
Table 5. Multiple regression analysis on average $\mathrm{HbA1c}$, SD of $\mathrm{HbA1c}$ and $\mathrm{CV}$ of $\mathrm{HbA1c}$.

\begin{tabular}{|c|c|c|c|c|c|c|}
\hline \multirow{2}{*}{ Male } & \multicolumn{2}{|c|}{ Average HbA1c } & \multicolumn{2}{|c|}{ SD of $\mathrm{HbA1c}$} & \multicolumn{2}{|c|}{$\mathrm{CV}$ of $\mathrm{HbA1c}$} \\
\hline & $\beta$ & $p$ & $\beta$ & $p$ & $\beta$ & $p$ \\
\hline Habitual miso consumption * & -0.086 & 0.290 & 0.017 & 0.817 & 0.028 & 0.721 \\
\hline Age, years & -0.201 & 0.025 & -0.116 & 0.146 & -0.113 & 0.140 \\
\hline Body mass index, $\mathrm{kg} / \mathrm{m}^{2}$ & 0.066 & 0.442 & 0.072 & 0.337 & 0.067 & 0.408 \\
\hline Exercise + & 0.099 & 0.232 & -0.061 & 0.397 & -0.065 & 0.412 \\
\hline Smoking $\ddagger$ & -0.006 & 0.940 & 0.0004 & 0.996 & -0.028 & 0.716 \\
\hline Insulin treatment $\S$ & 0.139 & 0.101 & 0.018 & 0.805 & 0.025 & 0.755 \\
\hline Duration of diabetes, years & 0.147 & 0.121 & -0.052 & 0.529 & -0.059 & 0.511 \\
\hline Average of $\mathrm{HbA} 1 \mathrm{c}, \%$ & - & - & 0.496 & $<0.001$ & 0.347 & $<0.001$ \\
\hline Intake of energy, $\mathrm{kcal} / \mathrm{kg}$ IBW/day & -0.061 & 0.512 & 0.168 & 0.042 & 0.172 & 0.054 \\
\hline $\begin{array}{c}\text { Intake of carbohydrate per energy } \\
\text { intake, } \%\end{array}$ & -0.063 & 0.431 & -0.061 & 0.382 & -0.049 & 0.520 \\
\hline Intake of dietary fiber, $\mathrm{g} /$ day & -0.038 & 0.685 & -0.082 & 0.317 & -0.081 & 0.358 \\
\hline \multirow{2}{*}{ Female } & \multicolumn{2}{|c|}{ Average HbA1c } & \multicolumn{2}{|c|}{ SD of HbA1c } & \multicolumn{2}{|c|}{ CV of $\mathrm{HbA1c}$} \\
\hline & $\beta$ & $p$ & $\beta$ & $p$ & $\beta$ & $p$ \\
\hline Habitual miso consumption * & -0.251 & 0.009 & -0.175 & 0.016 & -0.185 & 0.022 \\
\hline Age, years & -0.126 & 0.193 & -0.214 & 0.003 & -0.198 & 0.014 \\
\hline Body mass index, $\mathrm{kg} / \mathrm{m}^{2}$ & 0.059 & 0.533 & 0.073 & 0.297 & 0.057 & 0.464 \\
\hline Exercise $t$ & -0.163 & 0.056 & -0.128 & 0.045 & -0.137 & 0.052 \\
\hline Smoking $\ddagger$ & 0.138 & 0.108 & -0.089 & 0.161 & -0.082 & 0.244 \\
\hline Insulin treatment $\S$ & 0.048 & 0.586 & 0.064 & 0.327 & 0.091 & 0.214 \\
\hline Duration of diabetes, years & 0.192 & 0.044 & -0.098 & 0.169 & -0.097 & 0.217 \\
\hline Average of $\mathrm{HbA} 1 \mathrm{c}, \%$ & - & - & 0.572 & $<0.001$ & 0.494 & $<0.001$ \\
\hline Intake of energy, $\mathrm{kcal} / \mathrm{kg} \mathrm{IBW/day}$ & -0.007 & 0.948 & 0.011 & 0.886 & 0.012 & 0.889 \\
\hline $\begin{array}{c}\text { Intake of carbohydrate per energy } \\
\text { intake, } \%\end{array}$ & 0.028 & 0.738 & 0.036 & 0.569 & 0.013 & 0.847 \\
\hline Intake of dietary fiber, g/day & 0.094 & 0.399 & 0.014 & 0.865 & 0.034 & 0.711 \\
\hline
\end{tabular}

SD, standard deviation; CV, coefficient of variation; IBW, ideal body weight. * Habitual miso consumption was defined as non-habitual miso consumption $(=0)$ or habitual miso consumption $(=1)$. † Exercise was defined as non-regular exerciser $(=0)$ or regular exerciser $(=1)$. $\ddagger$ Smoking status was defined as non-smoker $(=0)$ or smoker $(=1)$. $§$ Insulin sensitizers, insulin secretagogues, insulin treatment and nutrient load reducers were defined as without $(=0)$ or with $(=1)$.

\section{Discussion}

In this study, we examined the relationship between habitual miso consumption and glycemic control in patients with T2DM and found that habitual miso consumption was independently related to glycemic control, including glycemic variability, which is reported to be a risk factor for microvascular and macrovascular complications in female with T2DM $[3,4,23]$. In this study, $88.1 \%$ of male and $82.3 \%$ of female were categorized as habitual miso consumption, which was higher than the general population as previously reported $[11,24]$. There is a possibility that people with T2DM might consume miso more usually than general people.

Fermentation is one of the main processes used to produce food from soybeans. During the production of fermented soybean products, bioactive constituents, including isoflavones and peptides, may alter their effectiveness in the treatment of T2DM [25]. The consumption of soybeans and fermented soybeans may be related to the lower prevalence of T2DM in Asians [26]. Various fermented soybean products are consumed in many Asian countries, including Japan, Korea, China, Indonesia, and Vietnam. In Japan, the most popular fermented soybean products are miso, natto, and soy sauce. Miso suppresses the development of various diseases, and habitual miso soup consumption is related to reduced insulin resistance [8-11]. Nevertheless, the relationship between miso and glycemic control, including glycemic variability, has not been revealed in patients with T2DM.

The relationship between habitual miso consumption and glycemic control may be explained as follows: 
A previous study showed that fat accumulation in fat mass was suppressed by miso intake and clarified the anti-obesity effects of miso in mice model [27]. In fact, the consumption of miso soup is associated with lower insulin resistance in female [11]. Insulin resistance and visceral fat accumulation have been reported to be associated with glycemic control, including $\mathrm{HbA} 1 \mathrm{c}$ variability [5]. This suggests that habitual miso consumption is associated with glycemic parameters through reduced fat accumulation and insulin resistance, which may lower $\mathrm{HbA1c}$ variability.

Miso, which are rich in soybean-specific proteins, lecithin, isoflavones, saponin, lipids rich in polyunsaturated fatty acids, and vitamin $\mathrm{E}[7,28,29]$, is a product of fermented soybeans. Isoflavones may have beneficial effects for improving glucose homeostasis and diabetes [24]. Isoflavones are similar in structure to estradiol and so can combine to the vascular wall's estrogen receptor [30]. Moreover, soybean proteins and isoflavones have been reported to reduce visceral fat accumulation in animal models [31-33]. In addition, we revealed the association between habitual miso consumption and the abdominal obesity in a previous study [18]. Therefore, isoflavones in miso may have inhibited the accumulation of visceral fat and contributed to good glycemic control in female. A previous study revealed that $\beta$-conglycinin, which included in miso, was a beneficial food ingredient for prevention of obesity [34]. In addition, $\beta$-conglycinin was reported to decrease triglycerides levels [35,36]. Therefore, patients with habitual miso consumption may prevent visceral fat accumulation and improve glycemic control. However, there was no difference between triglycerides level of people with habitual miso consumption and that of people without in this study. This might be because that this study was an observational study; and thus, the physicians prescribed the medication for dyslipidemia, if the triglycerides level were high. Taken together, these findings suggest that females with habitual miso consumption may have better glycemic control.

Contrarily, habitual miso consumption was not related to glycemic control in male; however, the reason underlying this finding is unclear. Possible explanations might be that habitual miso consumption was not associated with diet quality in male. Consistent with our study, previous research showed a relationship of habitual miso soup consumption with insulin resistance in females, but not in males [11]. In addition, it was previously reported that soy intake may be related to a lower risk of T2DM in Japanese female, but not in Japanese male [37].

However, the limitations of this study should be shown. First, the frequency of miso soup intake was evaluated by self-reported data, and there was a possibility that the data was not accurate. Second, we did not assess the association between habitual miso consumption and insulin concentrations and postprandial blood sugar level in this study. We evaluated triglycerides to HDL cholesterol ratio, which is reported as a marker of insulin resistance [15], and found that there was no association between habitual miso consumption and triglycerides to HDL cholesterol ratio in this study $(0.8$ [0.5-1.6] vs. 0.8 [0.6-1.4], $p=0.924$ in male 0.7 [0.5-1.2] vs. 0.9 [0.6-1.2], $p=0.426$ in female). This might be because the effect of taking medication for dyslipidemia. Thus, it was not unclear that female with habitual miso consumption had lower insulin resistance than those without, and the association between habitual miso consumption and postprandial blood sugar level. Third, there is a possibility that different types of miso would affect the results, since several types of miso, such as rice koji miso, barley koji miso, soybean koji miso, and blended miso, were used in Japan. Unfortunately, however, we did not investigate type of miso in this study. Therefore, further research is needed the association between each type of miso glycemic variability. Fourth, because this was a cross-sectional study and the sample size was relatively small, we need further research, such as a study with more participants and a randomized clinical trial. Finally, this study included only Japanese; thus, it is unclear whether the results can be generalized to other ethnic populations. 


\section{Conclusions}

This study demonstrated the relationship between habitual miso consumption and glycemic variability in female, but not in male, with T2DM. Miso, one of the famous traditional Japanese food, has been showed that consumption of miso soup may help in the management of patients with T2DM.

Author Contributions: Conception the work: Y.H., A.K., R.S., A.M. and M.F. Design the work: F.T., Y.H., A.K., R.S., A.M., M.H. and M.F. Acquisition data: Y.H., A.K., R.S., A.M., T.O., E.U., N.K., S.M., T.S., H.O., N.N., M.H., M.A., M.Y. and M.F. Analysis and interpretation of data: F.T. and Y.H. Contribution discussion: A.K., R.S., A.M., T.O., E.U., N.K., S.M., T.S., H.O., N.N., M.H., M.A. and M.Y. Writing the manuscript: F.T. Revising the manuscript: Y.H. and M.F. All authors have read and agreed to the published version of the manuscript.

Funding: This work was supported by JSPS KAKENHI, Grant-in-Aid for Young Scientists [Grant Number 19K20187].

Institutional Review Board Statement: The study was conducted according to the guidelines of the Declaration of Helsinki, and approved by Ethics Committee of Kyoto Prefectural University of Medicine (No. RBMR-E-466-6).

Informed Consent Statement: Informed consent was obtained from all subjects involved in the study.

Data Availability Statement: The datasets generated during and/or analysed during the current study are available from the corresponding author on reasonable request.

Conflicts of Interest: Hashimoto reports personal fees from Mitsubishi Tanabe Pharma Corp, Novo Nordisk Pharma Ltd., Sanofi K.K., and Daiichi Sankyo Co. Ltd. outside the submitted work. Senmaru has received personal fees from MSD K.K., Sanofi K.K., Taisho Toyama Pharma Co., Ltd., Ono Pharma Co., Ltd., Mitsubishi Tanabe Pharma Co, Kowa Pharma Co., Ltd., Kyowa Hakko Kirin Co., Ltd., Eli Lilly Japan K.K., Takeda Pharma Co., Ltd., Kissei Pharma Co., Ltd., Astellas Pharma Inc., and Novo Nordisk Pharma Ltd., outside the submitted work. Ushigome received grant support from the Astellas Foundation for Research on Metabolic Disorders and the Japanese Study Group for Physiology and Management of Blood Pressure and received grant from Ono Pharma. Co., Ltd., Mitsubishi Tanabe Pharma Corp., and received personal fees from Novo Nordisk Pharma Ltd., Kowa Pharma Co. Ltd., AstraZeneca plc, Daiichi Sankyo Co. Ltd., Astellas Pharma Inc., Takeda Pharma Co., Ltd., Kyowa Kirin Co. Ltd., Nippon Boehringer Ingelheim Co. Ltd., Sumitomo Dainippon Pharma Co. Ltd., MSD K.K., and Taisho Toyama Pharma Co., Ltd. outside the submitted work. Hamaguchi reports grants from Sanofi K.K., Eli Lilly Japan K.K., Astellas Pharma Inc., Daiichi Sankyo Co. Ltd., Sumitomo Dainippon Pharma Co. Ltd., Takeda Pharma Co. Ltd., Novo Nordisk Pharma Ltd., Nippon Boehringer Ingelheim Co. Ltd., Mitsubishi Tanabe Pharma Corp, Asahi Kasei Pharma, and Kyowa Kirin Co. Ltd. outside the submitted work. Asano reports honoraria from Ono Pharmaceutical Co., Ltd., Abbott Japan Co., Ltd., AstraZeneca plc, Kowa Pharmaceutical Co., Ltd., Sumitomo Dainippon Pharma Co., Ltd., and Novo Nordisk Pharma Ltd. Yamazaki reports personal fees from, Kowa Pharma Co. Ltd., MSD K.K., Daiichi Sankyo Co. Ltd., AstraZeneca plc., Kowa Pharma Co. Ltd., Sumitomo Dainippon Pharma Co. Ltd., Kyowa Kirin Co. Ltd., Takeda Pharma Co. Ltd., and Ono Phama Co., Ltd. outside the submitted work. Prof. Fukui received grants from Astellas Pharma Inc., Tejin Pharma LtD., Kyowa Kirin Co., Ltd., Daiichi Sankyo Co. Ltd., Takeda Pharma Co. Ltd., MSD K.K., Kowa Pharma Co. Ltd., Novo Nordisk Pharma Ltd., Sanofi K.K., Kissei Phama Co. Ltd., Nippon Boehringer Ingelheim Co. Ltd., Ono Pharma Co. Ltd., Sanwa Kagagu Kenkyusho CO., LtD., Mitsubishi Tanabe Pharma Corp, Sumitomo Dainippon Pharma Co., Ltd., Nippon Chemiphar Co., Ltd., Abbott japan Co. Ltd., Taisho Pharma Co., Ltd., Eli Lilly Japan K.K., and Terumo Corp., and received honoraria from Kowa Pharma Co. Ltd., Sanofi K.K., Nippon Boehringer Ingelheim Co., Ltd., Novo Nordisk Pharma Ltd., Kyowa Kirin Co. Ltd., Sumitomo Dainippon Pharma Co. Ltd., Nipro Corp., Ono Pharma Co. Ltd., Teijin Pharma Ltd., Sanwa Kagaku Kenkyusho Co. Ltd., Mitsubishi Tanabe Pharma Corp., Daiichi Sankyo Co. Ltd., Medtronic Japan Co. Ltd., Johnson \& Johnson k.k. Medical Co., Astellas Pharma Inc., Taisho Pharma Co., Ltd., Arkray Inc., Bayer Yakuhin, Ltd., AstraZeneca K.K., Kissei Pharma Co., Ltd., Eli Lilly Japan K.K., Mochida Pharma Co. Ltd., Abbott japan Co. Ltd., MSD K.K., and Takeda Pharma Co. Ltd. outside the submitted work. The other authors have nothing to disclose. 


\section{References}

1. Turner, R. Intensive blood-glucose control with sulphonylureas or insulin compared with conventional treatment and risk of complications in patients with type 2 diabetes (UKPDS 33). Lancet 1998, 352, 837-853. [CrossRef]

2. Holman, R.R.; Paul, S.K.; Bethel, M.A.; Matthews, D.R.; Neil, H.A.W. 10-Year Follow-up of Intensive Glucose Control in Type 2 Diabetes. N. Engl. J. Med. 2008, 359, 1577-1589. [CrossRef]

3. Hirakawa, Y.; Arima, H.; Zoungas, S.; Ninomiya, T.; Cooper, M.; Hamet, P.; Mancia, G.; Poulter, N.; Harrap, S.; Woodward, M.; et al. Impact of visit-to-visit glycemic variability on the risks of macrovascular and microvascular events and all-cause mortality in type 2 diabetes: The ADVANCE trial. Diabetes Care 2014, 37, 2359-2365. [CrossRef]

4. Wan, E.Y.F.; Fung, C.S.C.; Fong, D.Y.T.; Lam, C.L.K. Association of variability in hemoglobin A1c with cardiovascular diseases and mortality in Chinese patients with type 2 diabetes mellitus-A retrospective population-based cohort study. J. Diabetes Complicat. 2016, 30, 1240-1247. [CrossRef] [PubMed]

5. Jang, J.Y.; Moon, S.; Cho, S.; Cho, K.H.; Oh, C.M. Visit-to-visit HbA1c and glucose variability and the risks of macrovascular and microvascular events in the general population. Sci. Rep. 2019, 9, 1-8. [CrossRef]

6. Gorst, C.; Kwok, C.S.; Aslam, S.; Buchan, I.; Kontopantelis, E.; Myint, P.K.; Heatlie, G.; Loke, Y.; Rutter, M.K.; Mamas, M.A. Long-term glycemic variability and risk of adverse outcomes: A systematic review and meta-analysis. Diabetes Care 2015, 38, 2354-2369. [CrossRef]

7. Watanabe, H.; Kashimoto, N.; Kajimura, J.; Kamiya, K. A miso (Japanese soybean paste) diet conferred greater protection against hypertension than a sodium chloride diet in Dahl salt-sensitive rats. Hypertens. Res. 2006, 29, 731-738. [CrossRef]

8. Watanabe, H. Beneficial biological effects of miso with reference to radiation injury, cancer and hypertension. J. Toxicol. Pathol. 2013, 26, 91-103. [CrossRef]

9. Kanda, A.; Hoshiyama, Y.; Kawaguchi, T. Association of lifestyle parameters with the prevention of hypertension in elderly Japanese men and women: A Four-Year followup of normotensive subjects. Asia Pac. J. Public Health 1999, 11, 77-81. [CrossRef]

10. Manzoni, M.S.J.; Rossi, E.A.; Carlos, I.Z.; Vendramini, R.C.; Duarte, A.C.G.O.; Dâmaso, A.R. Fermented soy product supplemented with isoflavones affected fat depots in juvenile rats. Nutrition 2005, 21, 1018-1024. [CrossRef] [PubMed]

11. Ikeda, K.; Sato, T.; Nakayama, T.; Tanaka, D.; Nagashima, K.; Mano, F.; Joo, E.; Fujimoto, S.; Takahashi, Y.; Kosugi, S.; et al. Dietary habits associated with reduced insulin resistance: The Nagahama study. Diabetes Res. Clin. Pract. 2018, 141, 26-34. [CrossRef]

12. Sakai, R.; Hashimoto, Y.; Ushigome, E.; Miki, A.; Okamura, T.; Matsugasumi, M.; Fukuda, T.; Majima, S.; Matsumoto, S.; Senmaru, T.; et al. Late-night-dinner is associated with poor glycemic control in people with type 2 diabetes: The KAMOGAWA-DM cohort study. Endocr. J. 2018, 65, 395-402. [CrossRef] [PubMed]

13. Hashimoto, Y.; Kaji, A.; Sakai, R.; Osaka, T.; Ushigome, E.; Hamaguchi, M.; Yamazaki, M.; Fukui, M. Skipping breakfast is associated with glycemic variability in patients with type 2 diabetes. Nutrition 2020, 71, 110639. [CrossRef]

14. Lemmens, H.J.M.; Brodsky, J.B.; Bernstein, D.P. Estimating ideal body weight-A new formula. Obes. Surg. 2005, 15, 1082-1083. [CrossRef]

15. Fukuda, Y.; Hashimoto, Y.; Hamaguchi, M.; Fukuda, T.; Nakamura, N.; Ohbora, A.; Kato, T.; Kojima, T.; Fukui, M. Triglycerides to high-density lipoprotein cholesterol ratio is an independent predictor of incident fatty liver; a population-based cohort study. Liver Int. 2016, 36, 713-720. [CrossRef]

16. Matsuo, S.; Imai, E.; Horio, M.; Yasuda, Y.; Tomita, K.; Nitta, K.; Yamagata, K.; Tomino, Y.; Yokoyama, H.; Hishida, A. Revised Equations for Estimated GFR From Serum Creatinine in Japan. Am. J. Kidney Dis. 2009, 53, 982-992. [CrossRef]

17. Kobayashi, S.; Honda, S.; Murakami, K.; Sasaki, S.; Okubo, H.; Hirota, N.; Notsu, A.; Fukui, M.; Date, C. Both comprehensive and brief self-administered diet history questionnaires satisfactorily rank nutrient intakes in Japanese adults. J. Epidemiol. 2012, 22, 151-159. [CrossRef]

18. Takahashi, F.; Hashimoto, Y.; Kaji, A.; Sakai, R.; Kawate, Y.; Okamura, T.; Kitagawa, N.; Okada, H.; Nakanishi, N.; Majima, S.; et al. Habitual miso (Fermented soybean paste) consumption is associated with a low prevalence of sarcopenia in patients with type 2 diabetes: A cross-sectional study. Nutrients 2021, 13, 72. [CrossRef]

19. Sakai, R.; Hashimoto, Y.; Hamaguchi, M.; Ushigome, E.; Okamura, T.; Majima, S.; Asano, M.; Yamazaki, M.; Fukui, M. Living alone is associated with visit-to-visit HbA1c variability in men but not in women in people with type 2 diabetes: KAMOGAWA-DM cohort study. Endocr. J. 2020, 67, 419-426. [CrossRef]

20. Kang, H.M.; Kim, D.J. Total energy intake may be more associated with glycemic control compared to each proportion of macronutrients in the korean diabetic population. Diabetes Metab. J. 2012, 36, 300-306. [CrossRef]

21. Katagiri, R.; Sawada, N.; Goto, A.; Yamaji, T.; Iwasaki, M.; Noda, M.; Iso, H.; Tsugane, S. Association of soy and fermented soy product intake with total and cause specific mortality: Prospective cohort study. BMJ 2020, 368, m34. [CrossRef] [PubMed]

22. Kanda, Y. Investigation of the freely available easy-to-use software "EZR" for medical statistics. Bone Marrow Transplant. 2013, 48, 452-458. [CrossRef] [PubMed]

23. Takao, T.; Suka, M.; Yanagisawa, H.; Matsuyama, Y.; Iwamoto, Y. Predictive ability of visit-to-visit variability in $\mathrm{HbA1c}$ and systolic blood pressure for the development of microalbuminuria and retinopathy in people with type 2 diabetes. Diabetes Res. Clin. Pract. 2017, 128, 15-23. [CrossRef] [PubMed]

24. Ito, K.; Miyata, K.; Mohri, M.; Origuchi, H.; Yamamoto, H. The Effects of the Habitual Consumption of Miso Soup on the Blood Pressure and Heart Rate of Japanese Adults: A Cross-sectional Study of a Health Examination. Intern. Med. 2017, 56, 23-29. [CrossRef] 
25. Kwon, D.Y.; Daily, J.W.; Kim, H.J.; Park, S. Antidiabetic effects of fermented soybean products on type 2 diabetes. Nutr. Res. 2010, 30,1-13. [CrossRef] [PubMed]

26. Bergendal, E. Legume and soy food intake and the incidence of type 2 diabetes in the Shanghai Women's Health Study. Bone 2008, 23, 1-7.

27. Okouchi, R.; Sakanoi, Y.; Tsuduki, T. Miso (Fermented soybean paste) suppresses visceral fat accumulation in mice, especially in combination with exercise. Nutrients 2019, 11, 560. [CrossRef]

28. Yoshinaga, M.; Toda, N.; Tamura, Y.; Terakado, S.; Ueno, M.; Otsuka, K.; Numabe, A.; Kawabata, Y.; Uehara, Y. Japanese traditional miso soup attenuates salt-induced hypertension and its organ damage in Dahl salt-sensitive rats. Nutrition 2012, 28, 924-931. [CrossRef] [PubMed]

29. Prevention by Long-Term Fermented Miso of Induction of Colonic Aberrant Crypt Foci by Azoxymethane in F344 Rats-PubMed n.d. Available online: https:/ / pubmed.ncbi.nlm.nih.gov/11748458/ (accessed on 6 November 2020).

30. Vitale, D.C.; Piazza, C.; Melilli, B.; Drago, F.; Salomone, S. Isoflavones: Estrogenic activity, biological effect and bioavailability. Eur. J. Drug Metab. Pharmacokinet. 2013, 38, 15-25. [CrossRef]

31. Aoyama, T.; Fukui, K.; Takamatsu, K.; Hashimoto, Y.; Yamamoto, T. Soy protein isolate and its hydrolysate reduce body fat of dietary obese rats and genetically obese mice (yellow KK). Nutrition 2000, 16, 349-354. [CrossRef]

32. Davis, J.; Higginbotham, A.; O'Connor, T.; Moustaid-Moussa, N.; Tebbe, A.; Kim, Y.C.; Cho, K.W.; Shay, N.; Adler, S.; Peterson, R.; et al. Soy protein and isoflavones influence adiposity and development of metabolic syndrome in the obese male ZDF rat. Ann. Nutr. Metab. 2007, 51, 42-52. [CrossRef]

33. Wu, J.; Wang, X.; Chiba, H.; Higuchi, M.; Nakatani, T.; Ezaki, O.; Cui, H.; Yamada, K.; Ishimi, Y. Combined intervention of soy isoflavone and moderate exercise prevents body fat elevation and bone loss in ovariectomized mice. Metabolism 2004, 53, 942-948. [CrossRef] [PubMed]

34. Kohno, M.; Hirotsuka, M.; Kito, M.; Matsuzawa, Y. Decreases in serum triacylglycerol and visceral fat mediated by dietary soybean $\beta$-conglycinin. J. Atheroscler. Thromb. 2006, 13, 247-255. [CrossRef] [PubMed]

35. Aoyama, T.; Kohno, M.; Saito, T.; Fukui, K.; Takamatsu, K.; Yamamoto, T.; Hashimoto, Y.; Hirotsuka, M.; Kito, M. Reduction by phytate-reduced soybean beta-conglycinin of plasma triglyceride level of young and adult rats. Biosci. Biotechnol. Biochem. 2001, 65, 1071-1075. [CrossRef] [PubMed]

36. Tachibana, N.; Iwaoka, Y.; Hirotsuka, M.; Horio, F.; Kohno, M. Beta-conglycinin lowers very-low-density lipoprotein-triglyceride levels by increasing adiponectin and insulin sensitivity in rats. Biosci. Biotechnol. Biochem. 2010, 74, 1250-1255. [CrossRef] [PubMed]

37. Nanri, A.; Mizoue, T.; Takahashi, Y.; Kirii, K.; Inoue, M.; Noda, M.; Tsugane, S. Soy product and isoflavone intakes are associated with a lower risk of type 2 diabetes in overweight Japanese women. J. Nutr. 2010, 140, 580-586. [CrossRef] 